\title{
New hope for old bones
}

Age-related bone loss in mice (a mimic of osteoporosis in the elderly) can be prevented by eliminating senescent cells or suppressing their senescence-associated secretory phenotype, according to new research from the Mayo Clinic. The findings - a result of collaboration between the laboratories of James Kirkland and Sundeep Khosla firmly establish a causal role for cellular senescence in the bone loss that occurs with ageing.

The investigators used a combination of genetic (caspase 8-mediated) and pharmacological approaches (senolytic drugs (dasatinib plus quercetin) or a Janus kinase inhibitor) to target senescent cells in old mice with established bone loss.
“Intervening in 20- to 22-month-old mice (the equivalent of $\sim 70$ years in humans) for 2-4 months with each of the three approaches resulted in higher bone mass and strength, and better bone architecture than in vehicle-treated mice," explains Khosla. "Furthermore, the beneficial skeletal effects of targeting senescent cells were due to lower bone resorption with either maintained (trabecular) or higher (cortical) bone formation compared with vehicle-treated controls."

The observed skeletal effects (both antiresorptive and anabolic) contrast sharply with those of current osteoporosis drugs, which inhibit bone resorption but concomitantly reduce bone formation. Moreover, as targeting senescence has been shown to both improve insulin sensitivity and cardiovascular function, and reduce frailty, the approach has the potential not only to improve bone mass but also to alleviate other age-related comorbidities. Having established proof of concept with this study, the Khosla and Kirkland laboratories plan to advance the dasatinib-quercetin combination (or other promising compounds currently under investigation) to early-phase clinical trials in humans.

David Holmes

ORIGINAL ARTICLE Farr, J. N. et al. Targeting cellular senescence prevents age-related bone loss in mice. Nat. Med. http://dx.doi.org/10.1038/ nm.4385 (2017)

FURTHER READING Farr, J. N. \& Khosla, S. Skeletal changes through the lifespan - from growth to senescence. Nat. Rev. Endocrinol. 11, 513-521 (2015) 